\title{
Salud colectiva: transformar percepciones profesionales para empoderar a la ciudadanía
}

Javier Mendizábal

Docente e investigador de la

Facultad de Ciencias de la Salud y

Servicio Social. Universidad Nacional

de Mar del Plata.
Universidad y salud /

Intervenciones

\section{Resumen}

Las enfermedades no trasmisibles y los determinantes de salud requieren superar el paradigma tradicional de la salud pública. Este paradigma continúa los caminos positivistas, de primacía clínica e infectológica, ponderada en la tecnología dura en un escenario de mercantilización y abuso de la industria farmacéutica. Los actores compiten en el campo de la salud gestando asimetrías de poder a través de acumulación de capitales. En contraposición, la salud colectiva se propone trabajar sobre la construcción de subjetividades de los actores sociales desde una perspectiva sociohistórica con relación a la salud y a su cuidado. De ese modo la comunidad se posiciona en el centro del cual parte la red de salud, se promueve el autocuidado y se motivan la actitud y aptitud crítica. Esta posibilidad transformadora de la realidad permite resignificar el derecho a la salud y cuestiona el sentido del derecho a estar sano. El presente trabajo abordará una experiencia sobre la Cátedra abierta de salud colectiva que se desarrolla en la Universidad Nacional de Mar del Plata.

Palabras clave

- promoción de la salud

- salud colectiva

- percepción profesional

- derecho a la salud

- empoderamiento de la comunidad

colectiva y derechos humanos. Se planifican espacios de debate comunitario en relación con el derecho a la salud y la problematización desde la salud colectiva.

En un contexto favorable para promover derechos y salud, desde la cátedra se celebra el continuo crecimiento que fortalece las luchas $y$ conservan el espíritu militante. Se propone compartir en adelante lo que en palabras de Michael Foucault (1994) es la agitación de nuestro propio pensamiento modificado y la modificación de los otros. Algunas de las actividades conceptualizadas son el resultado de aquello que emana a partir de la construcción sociohistórica de sus protagonistas.

\section{Prevención de Lesiones por Causas Externas (LCE): lo que nos mata y no se ve}

En Argentina, las LCE ocupan la primera causa de mortalidad entre el año de vida y los 35 años, y en algunas regiones hasta los 45. Según el Ministerio de Salud de la Nación, "las lesiones no intencionales, aquellas causadas por accidentes, dan cuenta de la mayor mortalidad en las primeras tres décadas de la vida, que cualquier otra causa de muerte en la Argentina" (2007:7). La mayoría de estas lesiones, según el boletín epidemiológico, corresponde a colisiones entre vehículos. Deberá comprenderse entonces la necesidad de articulación con otras áreas externas al Ministerio de Salud, como son las de los Ministerios de Educación, de Planificación y del Interior (que se ocupa, entre otras actividades, del trasporte público) que necesariamente deben contribuir a la educación y la promoción de la seguridad vial y de lesiones en general. Lugar especial y protagónico deberá jugar la rrera de Licenciatura en Servicio Social. 2) Entre ellos, al grupo de investigación "Promoción de la salud" de la Facultad de Ciencias de la Salud y Servicio Social de la Universidad de Mar del Plata. 


\section{(6)}

es necesario considerar además

los contextos en los cuales se desarrollan

las lesiones y cómo juega cada actor

de este campo de la salud

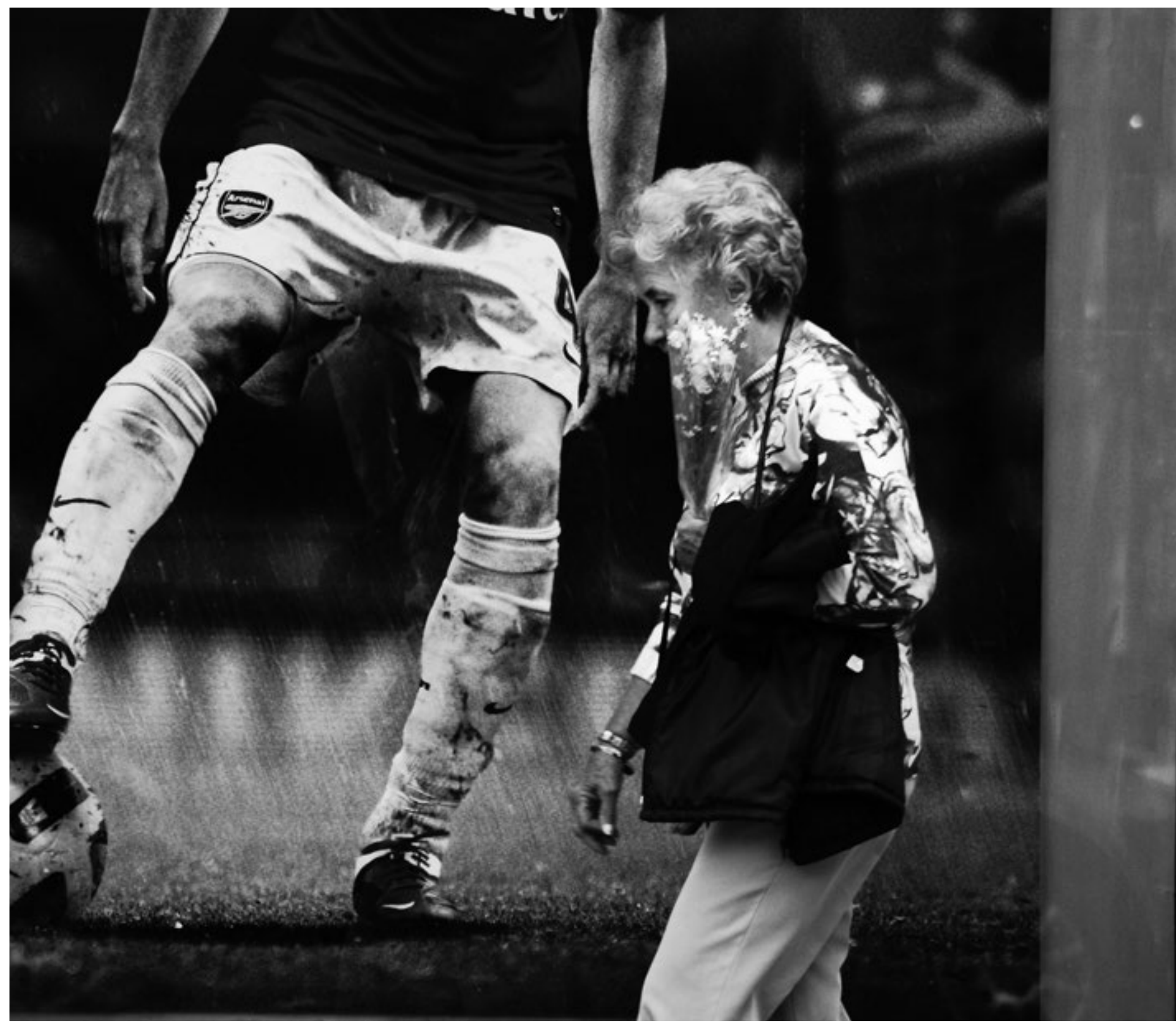


salud pública en la promoción de la salud a través de la atención primaria, de la formación de profesionales y de políticas coherentes al derecho a la salud reconocido en la Constitución Nacional y en declaraciones de derechos humanos. Para comenzar a transformar esta problemática hay que resignificar el concepto de "accidente" en "riesgos controlables y prevenibles" y así aumentar el valor simbólico comunitario en la transformación real del protagonismo y empoderamiento en incremento de salud colectiva.

En Argentina, el campo de salud se conforma bajo multiplicidad organizativa (Testa, 2005). El Estado es el que financia y da respuesta a la población que no posee cobertura por seguridad social o medicina prepaga. De todas formas, los hospitales públicos reciben, en gran parte del país, la totalidad de las lesiones en la vía pública para garantizar el acceso y la atención. A este punto es necesario reconocer el impacto económico de financiar los altos costos de las urgencias. La OMS calcula: "Los costos anuales estimados para los choques y lesiones causadas por el tránsito son alrededor del uno al dos por ciento (1 a 2 \%) del Producto Nacional Bruto (PNB)" (2004:3).

Esta situación exige responsabilidad en los actores involucrados y estrategias para implementar la promoción de salud, tales como la prevención de lesiones mediante leyes u ordenanzas (obligatoriedad de utilizar casco o cinturón, entre otras). La necesidad tanto de proteger la salud como derecho de la población como de interpretar el impacto económico compromete a garantizar el acceso a la salud y la calidad de la atención. La salud del trabajador se pone en riesgo ante la demanda de esfuerzo de trabajo que se reconoce prevenible (Morrone y Todisco, 2006). Según Spinelli:

"Con respecto al proceso de atención, el trabajo en salud se ve influenciado por el aumento en el número de usuarios, así como por el cambio en los perfiles demográficos y la heterogeneidad de patrones de vida de quienes acuden a los servicios de salud; esta heterogeneidad tiene que ver con asimetrías en el acceso a factores protectores, como educación, vivienda y políticas de promoción de salud" (2012:20).

Es necesario considerar además los contextos en los cuales se desarrollan las lesiones y cómo juega cada actor de este campo de la salud. En el partido de General Pueyrredón, provincia de Buenos Aires, no pueden escapar a este análisis las dificultades en el Servicio de Emergencias Médicas y la atención prehospitalaria; las dificultades actuales viales en relación con la corrupción en la entrega de licencias de conducir; el aumento demográfico y estival demandante de los servicios de salud; la precariedad histórica y conceptual de la atención primaria y la deficitaria articulación con los diferentes niveles de atención en gestión; la comunicación interestatal (entre Nación, provincia, municipio), las planificaciones en emergencias y catástrofes ante eventos históricos (políticos, culturales, turísticos, etc.); la centralización de todos los servicios y otros aspectos que revisten importancia en la epidemiología crítica (Ungaro, 2013).

Las muertes son sólo una parte de esta problemática personal y familiar, ya que en los casos que las lesiones no generan muerte pero sí secuelas individuales (entiéndanse no sólo las físicas), éstas repercuten en la familia y en la comunidad. Esas secuelas generan capacidades diferentes que limitan el ejercicio laboral -y por lo tanto económico-, los aspectos afectivo y relacional -y por ende el social一, el productivo y otras áreas que se suman y que pueden afectar a un individuo o una familia de forma permanente.

Este desafío en el campo de la salud nos insta a romper estructuras en el cuidado de la población. La noción de Modelo Médico Hegemónico (Menéndez, 1985) presenta la ideología de atender los acontecimientos relacionados con la salud/enfermedad/atención/ cuidado a través de un prisma biologicista, ahistórico, sin contexto social, con eficacia pragmática, individualista y mercantilista. Esta forma de entender y ejercer por parte de los profesionales de la salud impide dar respuestas reales a la problemática de las LCE. Es importante señalar que esto puede ser trabajado desde la extensión en propuestas desde un nuevo paradigma como el que propone la CASC, pero es sólo una parte. El mencionado modelo reproduce visiones, conceptos y prácticas en las que se mantiene la subalternidad de la sociedad. En contraposición, los proyectos que trabajan desde la óptica de la salud colectiva tienen un fuerte compromiso en cuanto a empoderar a la comunidad acerca del propio potencial a través de alternativas de autoatención ante cualquier padecimiento.

La CASC critica los viejos paradigmas asistencialistas y propone, mediante el análisis de un campo complejo de la salud, la relevancia de la participación activa de la comunidad para multiplicar la capacidad preventiva y el empoderamiento de la población. Así, propone para las LCE:

- Rechazar causas únicas como responsables para identificarlas con la comunidad a través de la epidemiología crítica y emplear el Método Altabir de planificación popular (Matus, 2007).

- Analizar al azar como mala fortuna o castigo divino devenidos en accidente y visibilizar las lesiones como fenómenos prevenibles.

- Criticar las conductas temerarias vinculadas a la masculinidad (Esteban et al., 2010) y pasar de la contemplación del riesgo a la actitud y aptitud preventiva y a la promoción de este ejercicio como estrategia de salud pública (Testa, 2007).

Esta mirada, en conjunto con otras acciones de mediano y largo plazo, permite, a muy bajo costo, observar la realidad y enunciarla desde otra perspectiva, posibilitando la transformación cognitiva y significativa del rol comunitario activo frente a las lesiones externas. Hugo Spinelli (2010) propone buscar respuestas a nuevas preguntas por medio de la acción en terreno y generar juego entre los actores que disputan capitales diferentes. En nuestro caso, se trata de ceder capital cultural para el aumento del capital simbólico comunitario y de obtener ganancias en el capital social y económico.

Se planifican entonces actividades que den relevancia al derecho a la salud. Así, la ética en salud pública se centra en el diseño y aplicación de medidas para la vigilancia y la mejora de la salud 
de las poblaciones. Este diseño demanda actores formados y comprometidos ideológicamente. En este sentido, Coleman, Bouësseau y Reis señalan:

"Asimismo, la ética de la salud pública trasciende la atención sanitaria para considerar las condiciones estructurales que promueven o dificultan el desarrollo de sociedades sanas" (2008:1).

La promoción de la salud y la responsabilidad del Estado en diseñar políticas públicas que protejan la salud de la población ${ }^{3}$ son argumentados desde un paternalismo libertario (Faden y Shebaya, 2010) frente al avance en la autonomía de la población. Dentro de las argumentaciones éticas, el paternalismo intenta justificar las intromisiones del Estado frente a los derechos individuales. El Paternalismo Puro (PP) y el Mixto (PM) difieren en las justificaciones que los sostienen. Según Faden y Shebaya (2010), las primeras se centrarán sólo en proteger a los individuos y las segundas tendrán más de una justificación: se busca cuidar a los individuos y/o a la comunidad y/o al ambiente y/o a los animales, por ejemplo. La semejanza entre ellas, y frente al paternalismo libertario $(\mathrm{PL})$, es que las primeras se mencionan como coacciones, con lo cual se impide la voluntad de quien es objeto. Podría señalarse además que estos tres tipos de paternalismos tendrán diferentes cargas de adaptación para los individuos. Así, en relación con las LCE causadas por tránsito, por ejemplo, el PP exige el uso de cascos para conducir un motovehículo como política pública, lo cual es una aparente carga para los conductores que sienten un atropello a su derecho a circular como deseen. El PM impide conducir con niveles de alcohol en sangre superiores a $0,5 \mathrm{~g} / \mathrm{l}$. Esta medida protege a conductores y peatones y permite conducir a quienes tengan menos de este límite. El PL promueve el uso de bicicleta a través de priorizar el paso y diseño de bicisendas y de mejorar el trasporte público. En este caso no hay carga ya que no habrá restricciones al acceso ni una participación obligatoria del ciudadano.

Abordar de forma crítica y analítica las LCE posibilita dimensionar la problemática que afecta a la población e interfiere en el derecho a la salud. La formación de profesionales de la salud que redimensione las problemáticas requiere esfuerzos y compromiso para estar a la altura del desafío que implica disminuir la mortalidad y la morbilidad de las LCE. A partir de esta premisa, desde la cátedra se implementaron en escuelas Centros de Atención Primaria de la Salud; salas de espera en las guardias de los hospitales, en sociedades de fomento, clubes; talleres para docentes, otros trabajadores, niños y cooperativistas, tales como: "Prevención de lesiones infectocontagiosas en la atención prehospitalaria en estudiantes a guardavidas/socorristas"; "Prevención de lesiones en programas comunitarios de huertas"; "Prevención de lesiones en el ámbito doméstico en el barrio Centenario en la ciudad

3) No sólo en LCE. Son ejemplos de gran intervención en la última década el tabaquismo y la obesidad. de Mar del Plata"; "Prevención de lesiones por intencionalidad: violencia bajo el programa de salud sexual y procreación responsable en un centro de atención primaria"; "Prevención de lesiones en escuelas primarias de la ciudad de Mar del Plata"; "Prevención de lesiones: intercambio de experiencias y conocimientos con estudiantes de pregrado de la carrera en Enfermería en el Hospital Interzonal Materno Infantil de la ciudad de Mar del Plata". Estas actividades planificadas junto a los estudiantes y supervisadas por los integrantes de la CASC posibilitaron aplicar un nuevo marco ideológico, se compartió el conocimiento con intención paternalista y se estimuló a los receptores de los talleres a que los difundan y repliquen en otros ámbitos, particularmente en sus hogares. La conciencia colectiva y la comprensión del trabajo de todos los actores de la sociedad es, además, indispensable para transformar la realidad. El pulso vital para la construcción del autocuidado en la comunidad parte del empoderamiento facilitado en la retroalimentación, la motivación y construcción ideológica en ese sentido es tan necesaria como desnaturalizar el accidente y concentrar el esfuerzo de trabajo en los problemas de la verdadera agenda del Estado.

\section{Bibliografía}

Coleman, C.; Bouësseau, M.; Reis, A. (2008). "Contribución de la ética a la salud pública", Boletín de la Organización Mundial de la Salud, Vol. 86, DOI: 577-656. Esteban, M. L. (2010). Antropología, género, salud y atención. Barcelona: Bellaterra. Faden, R.; Shebaya, S. (2010). "Public Health Ethics". En Edward N. Zalta (ed.) The Stanford Encyclopedia of Philosophy.

Foucault, M. (1994). Hermenéutica del Sujeto. Madrid. Endymión. Matus, C. (2007). MAPP Método Altabir de planificación popular. Buenos Aires: Lugar Editorial, Serie Salud Colectiva - UNLa.

Menéndez, E. (1985). "Modelo médico hegemónico, crisis socioeconómica y estrategias de acción del sector salud". En Cuadernos Médicos Sociales № 33, 3-34. Rosario. Ministerio de Salud de la Nación (2007). Boletín epidemiológico periódico: Lesiones por causas externas. Edición Especial.

Morrone, B.; Todisco, E. (2006). "Procesos destructivos y Procesos protectores en la práctica cotidiana de los profesionales de las ciencias de la salud". En Revista Argentina de Enfermería. RAE, N³6, 21-27. Córdoba.

Organización Mundial de la Salud (2004). Informe mundial sobre prevención de los traumatismos causados por el tránsito. Ginebra.

Spinelli, H. (2010). Las dimensiones del campo de la salud en Argentina. En revista Salud Colectiva, Vol. 6, №3, 275-293.

Spinelli, H. y otros (2013). La salud de los trabajadores de la salud. Trabajo, empleo, organización y vida institucional en hospitales públicos del aglomerado Gran Buenos Aires, Argentina, 2010-2012. Buenos Aires: OPS.

Testa, M. (2007). “Decidir en salud: ¿Quién?, ¿Cómo? y ¿Por qué?”. En revista Salud Colectiva, Vol. 3, № 3, 247-257.

Testa M. y colab. (2005). Pensar en Salud. Tercera edición. Buenos Aires: UNLa. Ungaro, J.; Úbeda, C.; Perinetti, A. (septiembre de 2013). "Lesiones por tránsito en el Partido Bonaerense de General Pueyrredón: Epidemiología, factores de riesgo y letalidad". En Revista Argentina de Salud Pública, Vol. 4, № 16. 